\title{
Numerical constraints and non-spatial open boundary conditions for the Wigner equation
}

\author{
Robert Kosik $^{1}$ D J Johann Cervenka ${ }^{1} \cdot$ Hans Kosina ${ }^{1}$
}

Received: 19 June 2021 / Accepted: 29 September 2021 / Published online: 3 November 2021

(c) The Author(s) 2021

\begin{abstract}
We discuss boundary value problems for the characteristic stationary von Neumann equation (stationary sigma equation) and the stationary Wigner equation in a single spatial dimension. The two equations are related by a Fourier transform in the nonspatial coordinate. In general, a solution to the characteristic equation does not produce a corresponding Wigner solution as the Fourier transform will not exist. Solution of the stationary Wigner equation on a shifted $k$-grid gives unphysical results. Results showing a negative differential resistance in IV-curves of resonant tunneling diodes using Frensley's method are a numerical artefact from using upwinding on a coarse grid. We introduce the integro-differential sigma equation which avoids distributional parts at $k=0$ in the Wigner transform. The Wigner equation for $k=0$ represents an algebraic constraint needed to avoid poles in the solution at $k=0$. We impose the inverse Fourier transform of the integrability constraint in the integrodifferential sigma equation. After a cutoff, we find that this gives fully homogeneous boundary conditions in the non-spatial coordinate which is overdetermined. Employing an absorbing potential layer double homogeneous boundary conditions are naturally fulfilled. Simulation results for resonant tunneling diodes from solving the constrained sigma equation in the least squares sense with an absorbing potential reproduce results from the quantum transmitting boundary with high accuracy. We discuss the zero bias case where also good agreement is found. In conclusion, we argue that properly formulated open boundary conditions have to be imposed on non-spatial boundaries in the sigma equation both in the stationary and the transient case. When solving the Wigner equation, an absorbing potential layer has to be employed.
\end{abstract}

Keywords Wigner equation · Neumann-Liouville equation · Characteristic von Neumann equation · Sigma equation · Quantum Liouville equation · Stationary $\cdot$ Algebraic constraint $\cdot$ Constrained Wigner equation · Open boundary conditions $\cdot$ Absorbing potential $\cdot$ Zero bias case $\cdot$ Non-spatial boundary

\section{Introduction}

Technology computer-aided design (TCAD) uses simulations to aid in semiconductor product development. Quantum transport models are indispensable to appropriately model modern devices and will further gain in relevance. The Wigner function method (WFM) [3, 24, 25] is based on a mathematical formulation of quantum mechanics which is

Robert Kosik

kosik@iue.tuwien.ac.at

Johann Cervenka

cervenka@iue.tuwien.ac.at

Hans Kosina

kosina@iue.tuwien.ac.at

1 Institute for Microelectronics, TU Wien, 1040 Vienna, Austria formally close to a classical phase space description [5, 26]. This allows for flexible mixed quantum-classical models and makes it an attractive approach for many applications where only parts of the system need to be modeled fully quantum mechanically. For example, the Wigner function approach can incorporate classical boundary conditions (BCs), semiclassical scattering models or classical force terms for the smooth part of the potential.

Quantum electron transport in modern semiconductor devices is described by a Wigner equation which is formally similar to the classical Liouville equation (also called Vlasov equation) [6]. Originally, the Wigner equation was used to simulate resonant tunneling diodes (RTDs) $[4,21]$. Now that the active region of transistors is getting closer and closer to the de Broglie wavelength, quantum mechanical effects already affect many currently manufactured devices. Several practical applications of the 
Wigner function method are demonstrated in the monograph [17].

\section{Quantum distribution functions}

Many key performance indicators of semiconductor devices are extracted from measured current-voltage characteristics. Those characteristics can be simulated by the numerical solution of stationary partial differential transport equations.

Stationary quantum transport is described by the Liouville-von Neumann equation for the density function $\rho(x, y)$

$-\frac{\hbar^{2}}{2 m}\left(\frac{\partial^{2}}{\partial x^{2}}-\frac{\partial^{2}}{\partial y^{2}}\right) \rho+(V(x)-V(y)) \rho=0$.

Here, $V(x)$ is the potential energy. We will always assume constant mass $m$ and only deal with a single spatial dimension in this paper. From the density function $\rho(x, y)$, the Wigner function $f(r, k)$ is defined as the result of two consecutive transformations described below.

\subsection{Sigma function (characteristic function)}

First, we introduce new coordinates for the quantum density

$r=\frac{x+y}{2}, \quad s=x-y$.

Using these coordinates, the density matrix transforms into the sigma function

$\sigma(r, s)=\rho\left(r+\frac{s}{2}, r-\frac{s}{2}\right)$

and the stationary von Neumann equation transforms into the stationary sigma equation

$\frac{\hbar^{2}}{m} \frac{\partial^{2} \sigma}{\partial r \partial s}=U(r, s) \sigma(r, s)$

where the potential term $U(r, s)$ is defined by

$U(r, s)=V\left(r+\frac{s}{2}\right)-V\left(r-\frac{s}{2}\right)$.

In a single spatial dimension, Eq. (3) is the characteristic hyperbolic form of the stationary von Neumann Eq. (1). The sigma function has the symmetry property

$\sigma(r,-s)=\overline{\sigma(r, s)}$.

Its real part is an even function, the imaginary part is an odd function of $s$.
Particle density and local current are given as

$n(r)=\sigma(r, 0), \quad j(r)=-\left.\frac{i \hbar}{m} \frac{\partial}{\partial s} \sigma(r, s)\right|_{s=0}$.

For practical simulation, a numerical cutoff parameter $L$ in $s$-space has to be introduced which restricts $s$ to an interval $[-L, L]$ symmetric around $s=0$. This cutoff parameter is called the coherence length and is also used in the Wigner equation.

\subsection{Wigner function}

The Wigner function $f(r, k)$ is then derived from the sigma function $\sigma(r, s)$ via a Fourier transform in coordinate $s$

$f(r, k)=\frac{1}{2 \pi} \int \sigma(r, s) e^{-i k s} \mathrm{~d} s$.

With these conventions, a wave function of the form $\psi(x)=e^{k_{0} x}$ has a corresponding sigma function $\sigma(r, s)=e^{k_{0} s}$ and a Wigner function $f(r, k)=\delta\left(k-k_{0}\right)$.

Applying the Fourier transform (7) to the sigma equation (3) gives the stationary Wigner equation

$\frac{\hbar k}{m} \frac{\partial f(r, k)}{\partial r}=\int f\left(r, k-k^{\prime}\right) V_{w}\left(r, k^{\prime}\right) \mathrm{d} k^{\prime}$.

Here, the Wigner potential $V_{w}(r, k)$ is defined as the Fourier transform of $U(r, s)$ divided by $i \hbar$

$V_{w}(r, k)=\frac{1}{\hbar} \frac{1}{2 \pi} \int U(r, s) e^{-i k s} \mathrm{~d} s$.

The Wigner potential is a real function odd in variable $k$. For non-zero bias, it has a $1 / k$-pole at $k=0$. Particle density and local current are given as

$n(r)=\int f(r, k) d k, \quad j(r)=\frac{\hbar}{m} \int k f(r, k) \mathrm{d} k$.

\subsection{Spatial boundary conditions}

For open systems, classical inflow boundary conditions are imposed on the stationary Wigner equation (two-point boundary value problem)

$f\left(r_{\min }, k\right)=f_{L}(k) \quad($ for $k>0)$

$f\left(r_{\max }, k\right)=f_{R}(k) \quad($ for $k<0)$.

Here, $f_{L}$ and $f_{R}$ are prescribed distributions in the left and right reservoir depending on temperature and the doping in the electrodes.

The existence and uniqueness of solutions to the stationary Wigner Eq. (8) with inflow boundary conditions is a 
long-standing open problem even in a single spatial dimension $[1,14,15]$.

\subsection{Formal classical limit}

For the classical limit of the sigma equation, we rescale the difference coordinate $s$ and introduce a new coordinate $\tau=s / \hbar$. We label the function in rescaled coordinates as $\tilde{\sigma}(r, \tau)=\sigma(r, \hbar \tau)$.

With $p=\hbar k$ we have $k s=p \tau$ and the Fourier transform of $\tilde{\sigma}(r, \tau)$ is $\tilde{f}(r, p)$. To perform the formal limit $\hbar \rightarrow 0$, we make the linear approximation

$U(r, s)=V\left(r+\frac{\hbar \tau}{2}\right)-V\left(r-\frac{\hbar \tau}{2}\right) \approx \hbar \tau V^{\prime}(r)$

The stationary sigma equation becomes in this formal limit

$\frac{1}{m} \frac{\partial}{\partial r} \frac{\partial}{\partial \tau} \tilde{\sigma}=-F(r) \tau \tilde{\sigma}$

where $F(r)=-V^{\prime}(r)$ is the force field. Equation (12) is the inverse Fourier transform of the stationary Vlasov equation

$\frac{p}{m} \frac{\partial \tilde{f}}{\partial r}+F(r) \frac{\partial \tilde{f}}{\partial p}=0$

\section{Numerical solution on a shifted $\boldsymbol{k}$-grid}

Frensley's original method [4] uses a shifted $k$-grid exluding the point $k=0$. Using a discrete Fourier transform, it is numerically much cheaper to solve for the solution in $s$-space where the equation is sparse in both coordinates, while the Wigner equation is not sparse in coordinate $k$. This has been undertaken in $[11,12]$ and is discussed below. We use shooting methods to reduce the two-sided problem (inflow boundary conditions) to the one-sided case.

\subsection{Integral form and Goursat problem}

Integrating both sides of Eq. (3) over a rectangular domain gives

$\sigma(R, S)=\sigma_{b}(R, S)+\int_{0}^{R} \int_{0}^{S} \frac{m}{\hbar^{2}} U(r, s) \sigma(r, s) \mathrm{d} r \mathrm{~d} s$.

The function $\sigma_{b}(r, s)$ is a solution to the homogeneous sigma equation

$\frac{\partial^{2} \sigma_{b}(r, s)}{\partial r \partial s}=0$

and is fixed by boundary conditions of Goursat type (BCs $\varphi(r), \psi(s)$ on two non-parallel characteristic lines forming an angle, see Fig. 1)

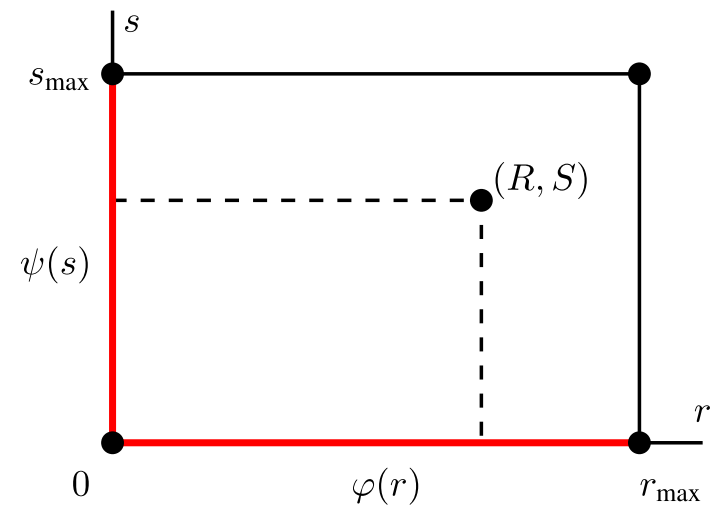

Fig. 1 Goursat boundary conditions are given on two non-parallel characteristic lines (angle)

$\sigma_{b}(r, s)=\varphi(r)+\psi(s)-\varphi(0), \quad \varphi(0)=\psi(0)$.

Equation (14) is a two-dimensional integral equation of Volterra type. Existence and uniqueness of the solution can be proved $[2,7,22]$.

We can arrive at a solution in full $s$-space by solving two Goursat problems separately in upper and lower half space where solutions are matched through the shared boundary condition $\phi(r)$. In general, these solutions will not be $L^{2}$ -integrable and the Fourier transform does not exist as an ordinary function.

There is a striking discrepancy between Goursat boundary conditions and the boundary conditions for the Wigner equation. In the Wigner equation, no boundary conditions corresponding to $\phi(r)$ are specified (however, compare the discussion in Sect. 6). The Wigner and the sigma equation are formally equivalent via Fourier transform. But existence of the Fourier transform as an ordinary function is a nontrivial condition, and for this reason, the set of solutions to both equations is not in a simple bijective correspondence. This makes the mathematical analysis subtle.

\subsection{Shooting methods}

The Wigner function on a shifted $k$-grid ( $2 N$ points) is related via a discrete Fourier transform to a discrete sigma function on a non-shifted $s$-grid ( $2 N+1$ points) plus antiperiodic boundary conditions in $s$-space [16].

The one-sided boundary value problem with spatial boundary conditions $\psi(s)$ for $\sigma(r, s)$ given on an $s$-line $\left(r_{0}, s\right)$ and anti-periodic boundary conditions $\sigma(r, L)=-\sigma(r,-L)$ can be reduced to the case of Goursat type boundary value problems by splitting $\sigma$ into even and odd part and solving two separate equation systems in half space. The odd part fulfills $\sigma^{o}(r, 0)=0$ and the even part fulfills $\sigma^{e}(r, L)=0$. Hence, the one-sided problem with anti-periodic boundary conditions is also well-posed. 
In the Wigner equation, usually two-sided inflow boundary conditions are given which can be imposed in the sigma equation via the discrete Fourier transform. Existence and uniqueness of the solution to the two-sided inflow problem has been proved in [1] for the discrete Wigner equation on a shifted $k$-grid. The same problem formulated in $s$-space is equivalent via the discrete Fourier transform and also well-posed.

The two-sided problem can be reduced to the solution of the one-sided problem using shooting methods (both for the sigma and the Wigner equation). In this way, no system matrix has to be stored, the method can easily be parallelized and numerical solution for very dense meshes is practical.

\subsection{Failure of Frensley's method}

The shooting method for the sigma equation with non-spatial anti-periodic boundary conditions was presented in [11]. No simulation results have been included in [11], as simulation results from the sigma equation did not reproduce the negative differential resistance expected in the simulation of IVcurves for resonant tunneling diodes and an implementation error could not be excluded.

The reason for the failure was not readily understood until further work was performed which is included in $[10,12]$. It turned out that simulation results which showed no negative differential resistance were actually numerically correct. In the case of the sigma equation, the mesh spacing $(\Delta r, \Delta s)$ can be repeatedly refined until the solution does no longer change significantly and a numerical limit is reached. If the discrete Wigner equation is solved without upwinding using the same meshing parameters, we immediately closely reproduce the solution from the sigma equation showing no negative differential resistance in the IV-curve.

In contrast, IV-curves calculated using Frensley's method show a wished-for negative differential resistance. However, in Frensley's method, upwinding is used which introduces a huge discretization error in variable $r$. In the numerical limit, the solutions with and without upwinding have to agree. When using upwinding, the limit solution is only reproduced if the mesh is further refined in coordinate $r$ as this reduces the discretization error. This was accomplished in [12] using shooting methods. With refinement in $r$, the solution with upwinding slowly converges towards the solution without upwinding. A very dense $r$-mesh has to be used. For illustration, the example from [12] (Fig. 2 in the original) is reproduced here in Fig. 2.

We conclude that the negative differential resistance shown in simulations of IV-curves for RTDs based on Frensley's method is nothing but a numerical artefact stemming from the use of upwinding on a coarse mesh. Furthermore, we noticed strong negative concentractions near $k=0$ in

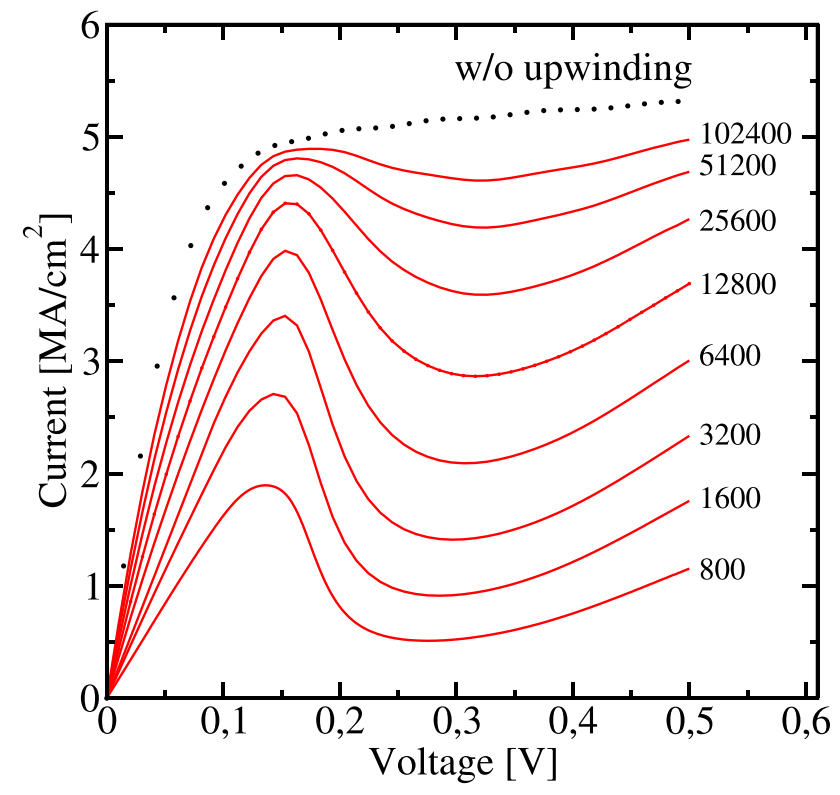

Fig. 2 The upper dotted black line represents the IV-curve for a RTD obtained from numerical solution of the Wigner equation without upwinding (resp. sigma equation with anti-periodic boundary conditions) calculated using $N_{r}=800$ points. The solid red lines are the IV-curves obtained from Frensley's discretization using upwinding. The grid is refined from $N_{r}=800$ up to $N_{r}=102400$. With refinement of $r$ the solutions slowly converge towards the solution without upwinding showing no negative differential resistance (Color figure online)

simulation results. It is these observations which motivated this work.

\section{Wigner equation at $k=0$}

In a single spatial dimension, Eq. (8) can be rewritten for $k \neq 0$ as

$$
\begin{aligned}
& \frac{\partial f(r, k)}{\partial r} \\
& \quad=\frac{1}{k} \frac{m}{\hbar} \int f\left(r, k-k^{\prime}\right) V_{w}\left(r, k^{\prime}\right) \mathrm{d} k^{\prime} \quad(\forall k \neq 0)
\end{aligned}
$$

The latter form emphasizes the fact that the Wigner equation becomes singular at $k=0$, which complicates its analysis. We can derive two equations for $k=0$ which are mathematically of different character:

1. Setting $k=0$ in Eq. (8) gives the following integrability constraint

$$
\int f\left(r,-k^{\prime}\right) V_{w}\left(r, k^{\prime}\right) \mathrm{d} k^{\prime}=0 .
$$


In this degenerate case, the left hand side in Eq. (8) vanishes and we do not get a differential equation. This special case is called an algebraic constraint in [1, 15] and is needed to avoid poles on the right hand side of Eq. (17). The integrability constraint has also a physical interpretation in the particle picture: The total potential inscattering rate at $k=0$ must vanish in the steady state.

2. If constraint (18) is fulfilled, then we can take the limit $k \rightarrow 0$ in Eq. (17). This gives the "transport" equation at $k=0$

$$
\frac{\partial f(r, 0)}{\partial r}=\frac{m}{\hbar} \int f_{k}\left(r,-k^{\prime}\right) V_{w}\left(r, k^{\prime}\right) \mathrm{d} k^{\prime}
$$

Here, $f_{k}(r, k)=\frac{\partial f(r, k)}{\partial k}$ denotes the first order derivative of $f(r, k)$ with respect to $k$. Alternatively, Eq. (19) can be derived by differentiating both sides of Eq. (8) with respect to $k$ and then setting $k=0$.

Derivation of the equations at $k=0$ assumes some regularity of $f(r, k)$, respectively, its partial derivatives near $k=0$, symbolically written as

$\left.k f_{r}(r, k)\right|_{k=0}=0$

$\left.k f_{r k}(r, k)\right|_{k=0}=0$

Two equations at $p=0$ can be derived also in the classical case. By setting $p=0$ in (13), we get

$\tilde{f}_{p}(r, 0) F(r)=0$

as the classical version of the quantum integrability constraint (18). Differentiating both sides of Eq. (13) with respect to $p$, we get the equation

$\tilde{f}_{r}(r, 0)=-m F(r) \tilde{f}_{p p}(r, 0)$

as the classical version of the transport Eq. (19).

The Wigner potential is odd in $k$, and in the stationary Wigner equation even and odd part only couple through inflow boundary conditions. The integrability constraint (18) only concerns the odd part of the Wigner function, while the transport equation at $k=0$ (19) only concerns the even part. As the (inverse) Fourier transform conserves parity, this parity analysis transfers to the sigma equation.

In this work, we use the quantum transmitting boundary method (QTBM) [13] for comparison with the Wigner function method. The Schrödinger modes occurring in the QTBM solution represent unnormalizable states. Their Wigner transform can be calculated analytically for simple potentials (e.g., potential step and barrier structures in 1D) and in general contains distributional parts. When summed up according to the distribution of incoming modes, the distributional parts are smoothed out and the resulting overall Wigner function does not contain distributional parts.

Here, the zero bias case (see Sect. 6) is exceptional because for each QTBM mode $\delta(k)$-singularities appear in its Wigner transform. Consequently, for zero bias, the Wigner transform of the full QBTM solution may contain distributional parts $\delta(k)$. For non-zero bias, the Wigner transform of each QTBM mode is found to be smooth at $k=0$ fulfilling both Eq. (18) and Eq. (19) in the appropriate sense.

\section{Constrained equation}

Solution of the Wigner equation on a shifted $k$-grid does not produce physically meaningful results as discussed in Sect. 3. Using a shifted $k$-grid, we can derive a discrete form of the transport equation at $k=0(19)$ by subtracting the discrete equations for $\Delta k / 2$ and $-\Delta k / 2$. But those solutions violate the integrability constraint (18).

A natural attempt to repair this is to include $k=0$ in the mesh which incorporates the integrability constraint. Using a suitable discrete inverse Fourier transform [16], this corresponds to periodic boundary conditions in $s$-space. Unfortunately, the sigma equation with periodic boundary conditions (and hence also the corresponding discrete Wigner equation) is ill-posed. This can be seen by a parity splitting: The odd part fulfills boundary conditions $\sigma^{o}(r, 0)=0$ and $\sigma^{o}(r, L)=0$ (overdetermined), while for the even part, we get no boundary conditions at all (underdetermined).

The equation for the even part can be made well-posed by imposing $\sigma^{e}(r, L)=0$. Together with $\sigma^{o}(r, L)=0$ this implies $\sigma(r, L)=\sigma(r,-L)=0$, i.e., double homogeneous boundary conditions. With double homogeneous boundary conditions, the sigma Eq. (3) is overdetermined and we refer to it as the constrained sigma equation.

Both periodic and anti-periodic boundary conditions are linked to the stationary Wigner equation at $k=0$ which is further discussed below.

1. The integrability constraint on a finite interval becomes

$$
\int_{-L}^{L} U(r, s) \sigma(r, s) \mathrm{d} s=0 .
$$

Integrating both sides of the sigma Eq. (3) over a finite $s$-interval $[-L, L]$ and using $(24)$

$$
\sigma_{r}(r, L)-\sigma_{r}(r,-L)=\frac{m}{\hbar^{2}} \int_{-L}^{L} U(r, s) \sigma(r, s) \mathrm{d} s=0
$$

results in periodic boundary conditions $\sigma_{r}(r, L)=\sigma_{r}(r,-L)$. 
2. Consider the stationary Wigner equation written in the singular form

$$
\frac{\partial f(r, k)}{\partial r}=\frac{1}{k} \frac{m}{\hbar} \int f\left(r, k-k^{\prime}\right) V_{w}\left(r, k^{\prime}\right) \mathrm{d} k^{\prime}
$$

Taking the inverse Fourier transform of Eq. (26) gives what we call the integro-differential sigma equation

$$
\frac{\partial \sigma(r, s)}{\partial r}=\frac{1}{2} \frac{m}{\hbar^{2}} \int \operatorname{sgn}\left(s-s^{\prime}\right) U\left(r, s^{\prime}\right) \sigma\left(r, s^{\prime}\right) \mathrm{d} s^{\prime}
$$

where sgn denotes the sign function.

In $s$-space there is a priori no unique antiderivative, the antiderivative is undefined by an additive constant. The derivative $\frac{\partial}{\partial s}$ turns into multiplication with $k$ in $k$-space. This operation has a canonical inverse operation in $k$-space, namely division by $k$, because we do not allow for distributional parts $\delta(k)$ in the Wigner function. This is tacitly assumed in Eq. (26).

By splitting the integral on the right hand side of Eq. (27)

$$
\begin{aligned}
H(s) & =\frac{1}{2} \int_{-\infty}^{\infty} \operatorname{sgn}\left(s-s^{\prime}\right) h\left(s^{\prime}\right) \mathrm{d} s^{\prime} \\
& =\frac{1}{2}\left[\int_{-\infty}^{s} h\left(s^{\prime}\right) \mathrm{d} s^{\prime}-\int_{s}^{\infty} h\left(s^{\prime}\right) \mathrm{d} s^{\prime}\right]
\end{aligned}
$$

we see that this expression picks out a specific antiderivative $H(s)$ of a function $h(s)$, in particular the arithmetic mean of upper and lower antiderivative.

Restricting the integro-differential sigma Eq. (27) to a finite $s$-domain $[-L, L]$

$$
\begin{aligned}
& \sigma_{r}(r, L)=\frac{1}{2} \frac{m}{\hbar^{2}} \int_{-L}^{L} U(r, s) \sigma(r, s) \mathrm{d} s \\
& \sigma_{r}(r,-L)=-\frac{1}{2} \frac{m}{\hbar^{2}} \int_{-L}^{L} U(r, s) \sigma(r, s) \mathrm{d} s
\end{aligned}
$$

results in anti-periodic boundary conditions $\sigma_{r}(r, L)=-\sigma_{r}(r,-L)$.

It follows that $\sigma_{r}=0$ and $\sigma$ is constant on the $s$-boundaries. The only reasonable choice for the integration constant is to set

$\sigma(r, L)=\sigma(r,-L)=0$

on a $s$-domain symmetric around $s=0$, i.e., fully homogeneous boundary conditions. Fully homogeneous boundary conditions have been motivated by inverse Fourier transform of the integrability constraint (18) and the transport equation at $k=0(19)$ in [10]. When solving the Wigner equation in $k$-space on a shifted $k$-grid, one has to incorporate the integrability constraint (18) which also results in an overdetermined system (constrained Wigner equation).

\section{Zero bias case}

It has to be pointed out that the zero bias case is exceptional. At zero bias, independent of the energy, the Wigner function resulting from a single Schrödinger mode (as used in the QTBM method) has a singularity at $k=0$ which stems from the long range correlation between the reflected and the transmitted part of the mode.

All the Wigner functions from the stationary Schrödinger modes contain distributional parts at $k=0$ which are not smoothed out when modes are summed up. If distributional parts at $k=0$ are allowed in the solution, then multiple solutions may actually exist.

A related kind of critique has been raised in the papers [18] and [23]. The authors claim a "failure of conventional boundary conditions" and suggest that the inflow boundary value problem might have multiple solutions. We note that those papers fail to identify the more fundamental cause of numerical problems (proper non-spatial open boundary conditions need to be employed, see Sect. 8).

Specific examples discussed by [18] use a symmetric and periodic potential, hence zero bias. In the zero bias case, the Wigner potential $V_{w}(r, k)$ is not singular at $k=0$ and we may allow for $\delta(k)$-terms and also for $1 / k$-poles in the solution $f(r, k)$. Using the ansatz

$f(r, k)=c(r) \delta(k)+\frac{h(r, k)}{k}$

in the Wigner Eq. (8), we get the Wigner equation for $h(r, k)$

$\frac{\partial h(r, k)}{\partial r}=c(r) \tilde{V}_{w}(r, k)+\int \frac{h\left(r, k-k^{\prime}\right)}{k-k^{\prime}} \tilde{V}_{w}\left(r, k^{\prime}\right) \mathrm{d} k^{\prime}$.

Here, we have defined $\tilde{V}_{w}=\frac{m}{\hbar} V_{w}$. In the ansatz (32) the function $c(r)$ can be freely chosen and acts like a boundary condition on the line $k=0$ (i.e., an analogue of Goursat type boundary data). The integrability constraint (18) will in general not be fulfilled by the solution if we allow for poles $1 / k$.

In the zero bias case, the solution to the stationary Wigner equation is non-unique. Here, the right choice of a functional space could help to filter out the relevant solution for the boundary value problem. Our numerical strategy is to assume an equilibrium distribution function without distributional parts or poles at $k=0$, and in this work, we always impose the constrained sigma equation. 


\section{Simulation results}

The constrained Wigner equation has first been introduced in [12]. Solving the constrained sigma equation in a least squares sense gives reasonable but not completely satisfactory results [10]. The natural way to incorporate double homogeneous boundary conditions is by employing an absorbing potential layer as recently introduced in [20]. This has the effect of damping down the solution near the non-spatial boundary. The imaginary potential is chosen as an even function in $s$ and we still get a real Wigner potential which can be employed when solving the Wigner equation. The absorbing potential couples even and odd parts of the equation inside the simulation domain which otherwise only couple through inflow boundary conditions.

In [8], a spectral method for the constrained sigma equation with an absorbing potential layer has been presented. This amounts to a discrete solution of the constrained Wigner equation in $k$-space. Memory requirements are huge and a special direct linear solver had to be implemented for the solution.

Below we present simulation results from solving the constrained sigma equation (double homogeneous boundary conditions) with an absorbing potential layer using finite differences. The corresponding discrete equation is sparse in both variables and memory requirements are much lower than if solving in $k$-space. The aim in this work is to investigate whether the constrained equation reproduces the solution from the QTBM in the numerical limit (fine mesh).

We use an orthogrid symmetric around $s=0$ including $s=0$ and a stencil

$$
\begin{gathered}
\sigma(0,0)+\sigma(1,1)-\sigma(0,1)-\sigma(1,0) \\
=\frac{1}{4} \frac{m}{\hbar^{2}} \sum_{i=0}^{1} \sum_{j=0}^{1} U(i, j) \sigma(i, j)
\end{gathered}
$$

for the unit square.

Given $\sigma$ on the boundary, we calculate $f$ on the boundary via the discrete Fourier transform and then impose inflow boundary conditions exactly as in Frensley's method on a shifted $k$-grid. As we are solving an overdetermined system, there is a freedom to choose equations which are imposed as strict constraints and which equations are only fulfilled in a least squares sense. In our over-determined method, the inflow boundary conditions are imposed as strict constraints.

The overdetermined system is then solved in a least squares sense with constraints exactly fulfilled. Mathematically this represents a quadratic programming problem with linear equality constraints. For the solution, we

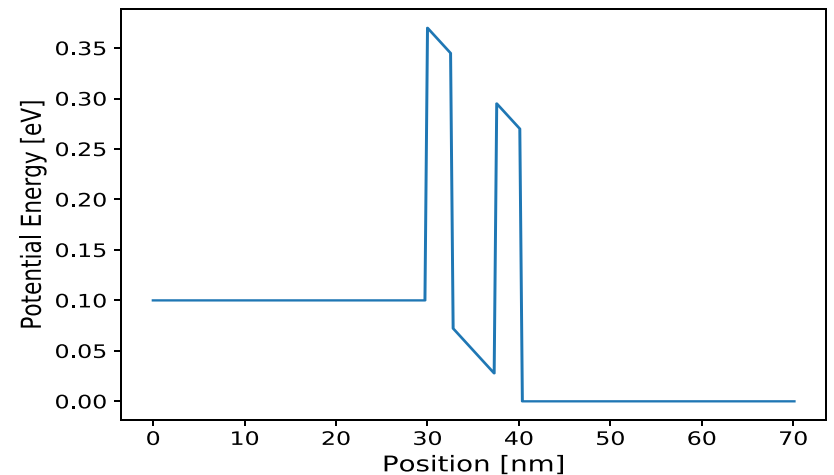

Fig. 3 Structure of the resonant tunneling diode with two AlGaAs barrier layers as used in simulations. In the plotted example, a bias of $-0.1 \mathrm{~V}$ is assumed

use the method described in [8] which avoids the explicit building of normal equations.

The numerical method is evaluated by simulating a GaAs/AlGaAs RTD and comparison with the quantum transmitting boundary method which we take as the reference model. The RTD structure used in the simulation is depicted in Fig. 3, it consists of a $4.5 \mathrm{~nm}$-wide GaAs quantum well and $2.8 \mathrm{~nm}$-wide AlGaAs barrier layers. The barrier height is $0.27 \mathrm{eV}$.

In order to get a good fit to QTBM a coherence length of at least $120 \mathrm{~nm}$ and a mesh spacing $\Delta r<0.1 \mathrm{~nm}$ should be employed. Here, we consider an even larger coherence length of $170 \mathrm{~nm}$ and a mesh size of $N_{r}=6000, N_{s}=5601$ with $\Delta s=2 \Delta r$. The length of the electrodes used in the simulation is $85 \mathrm{~nm}$ (half the coherence length). The coherence length and the mesh size have been chosen in order to study the method in the limit of a large coherence length and a fine mesh.

An absorbing layer is employed at upper and lower $s$-boundary. We use an imaginary quadratic potential with maximum value at the boundary corresponding to a bias of $2 \mathrm{~V}$. Each absorbing layer comprises $10 \%$ of half the $s$-domain.

Simulation results for particle density are depicted in Fig. 4. In general, the fit with QTBM is very good. For zero bias, we see a small deviation in the density at the center of the well but also in this case the fit is still good.

In order to demonstrate the numerical capability of the method, we simulate up to a high bias which is beyond the usual operating conditions of the device. In Fig. 5, we see a weak second resonance in the IV-characteristics for both QTBM and sigma function method. The fit between both methods is excellent. 


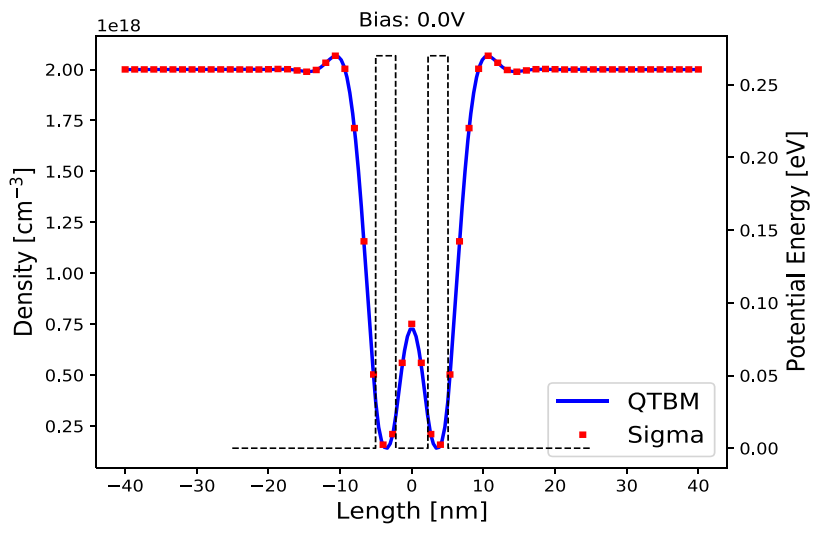

(a) $\operatorname{Bias}=0.0 \mathrm{~V}$

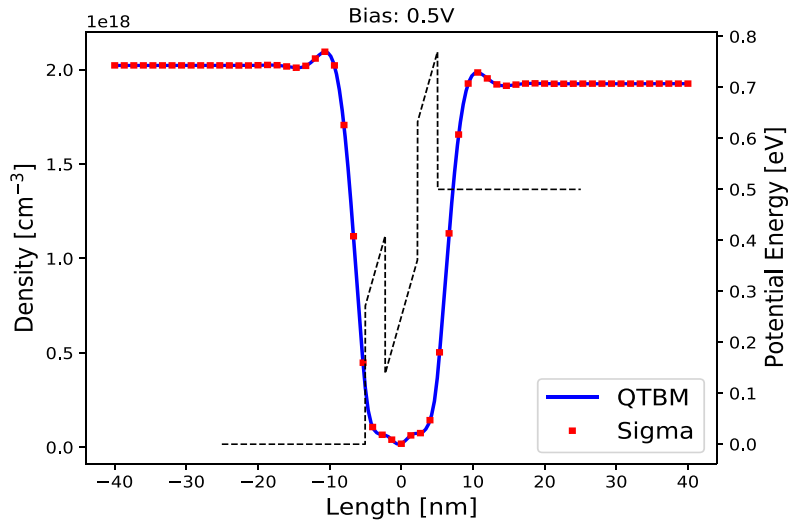

(b) $\operatorname{Bias}=0.5 \mathrm{~V}$

Fig. 4 Particle density in the active region of the RTD depicted in Fig. 3 for different biases comparing QTBM and constrained sigma equation. The dashed black lines indicate potential energy

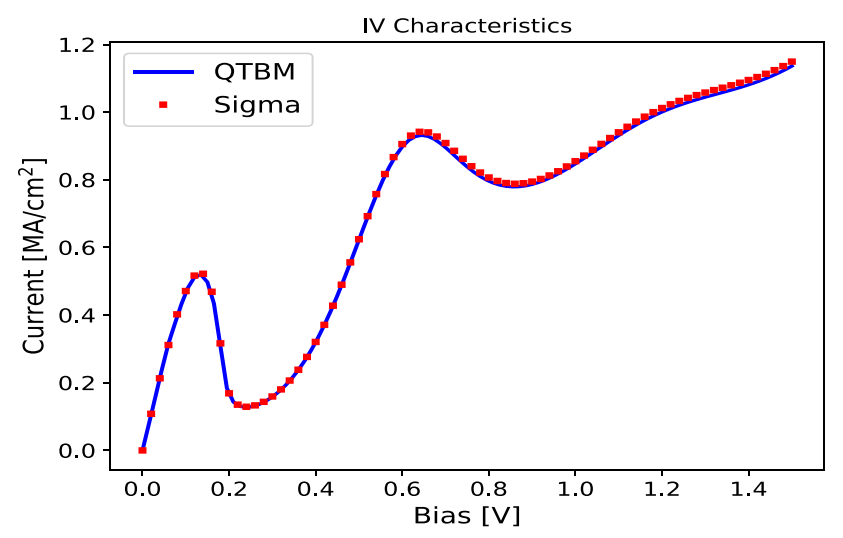

Fig. 5 Simulation of the current-voltage curve for the same resonant tunneling structure as used for Fig. 4. Again, good agreement between the solution based on the constrained sigma equation and the QTBM solution

\section{Conclusion}

Frensley's method [4] uses a shifted $k$-grid which ignores the integrability constraint (18). Results showing a negative differential resistance when simulating IV-curves of resonant tunneling diodes are an artefact of numerical diffusion (upwinding) using a too coarse mesh. The method breaks down in the limit of a fine mesh [10].

In order to avoid a singularity at $k=0$, the integrability constraint has to be incorporated when solving the stationary Wigner equation. The integro-differential sigma equation (27) avoids distributional parts in the Wigner transform at $k=0$. We impose the inverse Fourier transform of the integrability constraint in the integro-differential sigma equation. After a cutoff, double-homogeneous boundary conditions are derived.
To get results closely reproducing QTBM, an absorbing potential layer has to be employed. When using an absorbing potential, double homogeneous boundary conditions are naturally fulfilled and results from QTBM are reproduced with high accuracy. In the zero bias case, a solution to the stationary Wigner equation may contain distributional parts at $k=0$. Exemplary simulation results still show a good fit for the density $n(x)$ between QTBM and our method.

We want to stress that it is not necessary to actually solve an overdetermined system (neither for the sigma nor for the Wigner equation). Results presented in [20] demonstrate that combining an absorbing potential with antiperiodic boundary conditions gives good results closely reproducing results from QTBM. We have also experimented with combining an absorbing potential and periodic boundary conditions which again gives good results in numerical simulations but will not be further discussed here.

The overall insight from these studies is that proper open boundary conditions should be employed on non-spatial boundaries when solving the stationary sigma equation (characteristic Neumann equation). Analysis of the two equations for $k=0$ [10] has been a detour to arrive at this conclusion. An alternative formulation (not based on an absorbing potential and not overdetermined) of non-spatial open boundary conditions for the sigma equation has been suggested in [9].

We point out that the considerations above concern the stationary case as well as the transient case. Transient simulation of RTDs using an absorbing potential is demonstrated in [19]. In transient simulations, the absorbing potential avoids artificial reflections on non-spatial boundaries. The requirement to impose properly formulated open boundary conditions is in line with simulation 
of wave-like phenomena in various application domains, for example electromagnetic phenomena. Using an absorbing potential layer non-spatial open boundary conditions are conveniently incorporated in the transient/stationary Wigner equation.

Acknowledgements The financial support by the Austrian Science Fund (FWF): P33151 is gratefully acknowledged.

Funding Open access funding provided by TU Wien (TUW).

\section{Declarations}

Conflict of interest The authors declare that they have no conflict of interest.

Open Access This article is licensed under a Creative Commons Attribution 4.0 International License, which permits use, sharing, adaptation, distribution and reproduction in any medium or format, as long as you give appropriate credit to the original author(s) and the source, provide a link to the Creative Commons licence, and indicate if changes were made. The images or other third party material in this article are included in the article's Creative Commons licence, unless indicated otherwise in a credit line to the material. If material is not included in the article's Creative Commons licence and your intended use is not permitted by statutory regulation or exceeds the permitted use, you will need to obtain permission directly from the copyright holder. To view a copy of this licence, visit http://creativecommons.org/licenses/by/4.0/.

\section{References}

1. Arnold, A., Lange, H., Zweifel, P.F.: A discrete-velocity, stationary Wigner equation. J. Math. Phys. 41(11), 7167-7180 (2000). https://doi.org/10.1063/1.1318732

2. Brunner, H.: Collocation Methods for Volterra Integral and Related Functional Equations. Cambridge University Press, Cambridge (2004). https://doi.org/10.1017/cbo9780511543234

3. Ferry, D.K., Nedjalkov, M.: The Wigner Function in Science and Technology, pp. 2053-2563. IOP Publishing, Bristol (2018). https://doi.org/10.1088/978-0-7503-1671-2

4. Frensley, W.R.: Boundary conditions for open quantum systems driven far from equilibrium. Rev. Mod. Phys. 62(3), 745-791 (1990). https://doi.org/10.1103/RevModPhys.62.745

5. Gosson, M.A.D.: The Wigner Transform. Advanced Textbooks in Mathematics. World Scientific Publishing Company (EUROPE) (2017). https://doi.org/10.1142/q0089

6. Jacoboni, C., Bordone, P.: The Wigner-function approach to nonequilibrium electron transport. Rep. Prog. Phys. 67, 1033-1071 (2004). https://doi.org/10.1088/0034-4885/67/7/R01

7. Kharibegashvili, S.: Goursat and Darboux type problems for linear hyperbolic partial differential equations and systems. Memoirs Differ. Equ. Math. Phys. 4, 1-127 (1995)

8. Kosik, R., Cervenka, J., Kosina, H.: Numerical solution of the constrained Wigner equation. In: 2020 International Conference on Simulation of Semiconductor Processes and Devices (SISPAD). IEEE (2020). https://doi.org/10.23919/sispad49475.2020. 9241624

9. Kosik, R., Cervenka, J., Kosina, H.: Open boundary conditions for the Wigner and the characteristic von Neumann equation. In: Kim, K.Y., Weinbub, J., Everitt, M. (eds.) Book of Abstracts of the 4th International Wigner Workshop (IWW), pp. 42-43. Sejong,
Korea (2021). http://iww2021.sejong.ac.kr/include/file/IWW_ 2021_Book_of_Abstracts.pdf

10. Kosik, R., Cervenka, J., Thesberg, M., Kosina, H.: A revised Wigner function approach for stationary quantum transport. In: Large-Scale Scientific Computing, pp. 403-410. Springer International Publishing (2020). https://doi.org/10.1007/978-3-03041032-2 46

11. Kosik, R., Kampl, M., Kosina, H.: On the characteristic Neumann equation and the Wigner equation. In: Weinbub, J., Ferry, D.K., Knezevic, I., Nedjalkov, M., Selberherr, S. (eds.) Book of Abstracts of the 2nd International Wigner Workshop (IW2), pp. 26-27. Windermere, UK (2017). http://www.iue.tuwien.ac.at/ iw22017/wp-content/uploads/2017/06/IW2_Book_of_Abstracts_ print.pdf

12. Kosik, R., Thesberg, M., Weinbub, J., Kosina, H.: On the consistency of the stationary Wigner equation. In: Ferry, D.K., Weinbub, J., Goodnick, S. (eds.) Book of Abstracts of the 3rd International Wigner Workshop (IW2), pp. 30-31. Hilton Orrington Evanston, Illinois, USA (2019). https://www.iue.tuwien.ac.at/iwen2019/wpcontent/uploads/2019/06/IW2-2019-Book-of-Abstracts.pdf

13. Lent, C.S., Kirkner, D.: The quantum transmitting boundary method. J. Appl. Phys. 67(10), 6353-6359 (1990). https://doi. org/10.1063/1.345156

14. Li, R., Lu, T., Sun, Z.: Stationary Wigner equation with inflow boundary conditions: will a symmetric potential yield a symmetric solution? SIAM J. Appl. Math. 74(3), 885-897 (2014). https://doi. org/10.1137/130941754

15. Li, R., Lu, T., Sun, Z.: Parity-decomposition and moment analysis for stationary Wigner equation with inflow boundary conditions. Front. Math. China 12(4), 907-919 (2017). https://doi.org/10. 1007/s11464-017-0612-9

16. Martucci, S.A.: Symmetric convolution and the discrete sine and cosine transforms. IEEE Trans. Signal Process. SP-42, 10381051 (1994). https://doi.org/10.1109/78.295213

17. Querlioz, D., Dollfus, P., Mouis, M. (eds.): The Wigner Monte Carlo Method for Nanoelectronic Devices. Wiley, New York (2013). https://doi.org/10.1002/9781118618479

18. Rosati, R., Dolcini, F., Iotti, R.C., Rossi, F.: Wigner-function formalism applied to semiconductor quantum devices: Failure of the conventional boundary condition scheme. Phys. Rev. B 88(3), 035401.1-035401.16 (2013). https://doi.org/10.1103/physrevb.88. 035401

19. Schulz, L., Schulz, D.: Numerical analysis of the transient behavior of the non-equilibrium quantum Liouville equation. IEEE Trans. Nanotechnol. 17(6), 1197-1205 (2018). https://doi.org/ 10.1109/TNANO.2018.2868972

20. Schulz, L., Schulz, D.: Complex absorbing potential formalism accounting for open boundary conditions within the Wigner transport equation. IEEE Trans. Nanotechnol. 18, 830-838 (2019). https://doi.org/10.1109/TNANO.2019.2933307

21. Sun, J.P., Haddad, G., Mazumder, P., Schulman, J.: Resonant tunneling diodes: models and properties. Proc. IEEE 86(4), 641-660 (1998). https://doi.org/10.1109/5.663541

22. Suryanarayana, M.B.: On multidimensional integral equations of Volterra type. Pac. J. Math. 41(3), 809-828 (1972). https://doi. org/10.2140/pjm.1972.41.809

23. Taj, D., Genovese, L., Rossi, F.: Quantum-transport simulations with the Wigner-function formalism: failure of conventional boundary-condition schemes. Europhys. Lett. 74(6), 1060-1066 (2006). https://doi.org/10.1209/epl/i2006-10047-3

24. Weinbub, J., Ferry, D.K.: Recent advances in Wigner function approaches. Appl. Phys. Rev. 5(4), 041104 (2018). https://doi. org/10.1063/1.5046663

25. Wigner, E.P.: On the quantum correction for thermodynamic equilibrium. Phys. Rev. 40(5), 749-759 (1932). https://doi.org/ 10.1103/PhysRev.40.749 
26. Zachos, C., Fairlie, D., Curtright, T.: Quantum Mechanics in Phase Space-An Overview with Selected Papers. World Scientific, Singapore (2005). https://doi.org/10.1142/9789812703507
Publisher's Note Springer Nature remains neutral with regard to jurisdictional claims in published maps and institutional affiliations. 